Ebisu Ebisu

Études japonaises Études japonaises

50 | automne-hiver 2013

Création et valeurs dans le Japon moderne

\title{
L'ambivalence du récit de guerre : Mugi to heitai de Hino Ashihei, de l'expérience individuelle au récit commun
}

\author{
戦争文学の両義性一火野蔁平の『麦亡兵隊』、個人的な体騃力ら共 \\ 通の物語まで
}

The Ambivalence of War Accounts: Hino Ashihei's Mugi to Heitai, from

Individual Experience to Common Narrative

\section{Guillaume Muller}

\section{(2) OpenEdition}

Journals

Édition électronique

URL : http://journals.openedition.org/ebisu/1123

DOI : 10.4000/ebisu. 1123

ISSN : 2189-1893

Éditeur :

Institut français de recherche sur le Japon (UMIFRE 19 MAEE-CNRS), Maison franco-japonaise

Édition imprimée

Date de publication : 1 octobre 2013

Pagination : 69-94

ISSN : 1340-3656

Référence électronique

Guillaume Muller, «L'ambivalence du récit de guerre : Mugi to heitai de Hino Ashihei, de l'expérience individuelle au récit commun », Ebisu [En ligne], 50 | automne-hiver 2013, mis en ligne le 21 juin 2014, consulté le 20 avril 2019. URL : http://journals.openedition.org/ebisu/1123 ; DOI : 10.4000/ebisu.1123 


\title{
L'ambivalence du récit de guerre
}

\section{Mugi to heitai de Hino Ashihei, de l'expérience individuelle au récit commun}

\author{
Guillaume MULLER*
}

La publication en 1938 de Mugi to heitai 麦と兵隊 (Les Blés et les Soldats), le premier récit de guerre de Hino Ashihei 火野葦平 (1907-1960, de son vrai nom Tamai Katsunori 玉井勝則), fit de lui le plus célèbre des auteurs de littérature de guerre de son époque : Hino avait obtenu le sixième prix Akutagawa en 1937 pour un texte d'un tout autre genre ${ }^{1}$ alors qu'il était caporal d'infanterie en Mandchourie, et l'armée, consciente du potentiel que représentait le personnage en termes de propagande, le rattacha à son service d'Information (Hōdōbu 報道部). C'est à ce titre qu'il écrivit ce journal daté du 4 au 22 mai 1938, récit de la campagne de Xuzhou 徐州. Le texte fut d'abord publié en août 1938 dans la revue Kaizō 改造 avant d'être édité le mois suivant et se vendit à plus d'un million d'exemplaires. Hino s'imposa alors très rapidement au monde littéraire japonais avec une nouvelle façon d'écrire la guerre, fondée sur son statut de membre de l'armée. La publication de ce qu'il appelait " mes récits de guerre " (waga senki 我が戦記), dont Mugi to heitai constitue le premier volet, contrasta en effet vivement avec les écritures de la guerre qui prévalaient alors. Outre, évidemment, les articles des journalistes de métier dépêchés sur place, le

* INALCO, Centre d'études japonaises.

1. Le texte s'intitule Funnyōtan 粪尿譚 (Tas d'excréments) : cette nouvelle aux accents de littérature prolétarienne décrit comment des machinations politiques locales vont nuire au protagoniste, humble transporteur d'engrais du nom de Komori Hikotarō. 
domaine revenait d'une part aux écrivains envoyés sur le front quelques jours ou semaines par des journaux qui cherchaient une certaine littérarité, et d'autre part aux récits " authentiques " de soldats publiés dans la presse. Hino devint un personnage public ; en même temps que celui-ci accédait à la célébrité, le lectorat se forgea de lui une image qui définit le mode de réception de ses textes. Cette célébrité reposait en effet sur son statut de soldat, qui garantissait la crédibilité de sa prise de parole et, partant, son intérêt même : la population civile, en demande d'informations sur une guerre où un nombre conséquent de leurs proches étaient partis, et la propagande militaire, qui cherchait à créer un sentiment de proximité entre l'arrière et le front, avaient tous deux besoin de l'authenticité qu'offrait la présence de Hino sur le front.

Hino fut le premier et le plus célèbre représentant de cette nouvelle catégorie d'écrivains que l'on réunit communément sous le terme de " soldat-écrivain " (heitai sakka 兵隊作家), mais il ne fut pas le seul. Le potentiel, en termes de propagande (et probablement aussi de volume de ventes) qu'offraient les récits littéraires de soldats conduisit à l'apparition de textes produits dans des circonstances similaires sous la plume d'auteurs tels qu'Ueda Hiroshi 上田廣 et Hibino Shirō 日比野士郎 dont les premiers récits, Köjin 黄塵 (Nuage de poussière) et Goshō kurìku 呉淞クリーク (La crique de Wusong), parurent respectivement en novembre 1938 et juillet 1939. C'est dans une perspective similaire que le service des Renseignements du Cabinet (Naikaku jōhōbu 内閣情報部) créa, avec la collaboration active de Kikuchi Kan 菊池寛, les " unités de la plume » (pen butai ペン部隊). En septembre 1938, vingt-deux auteurs reconnus tels qu'Ozaki Shirō 尾崎士郎, Hayashi Fumiko 林芙美子, Yoshikawa Eiji 吉川英治 ou Niwa Fumio 丹羽文雄 partirent dans les zones de combat pour suivre l'armée quelques semaines et en tirer des textes réalistes sur les conditions de vie des soldats car « ce genre de tâches documentaires était nécessaire pour une excellente propagande, et à terme aidait à créer des liens entre l'armée et la société civile " (Kushner 2006 : 81). L'opération, répétée à plusieurs reprises ${ }^{2}$, n'eut cependant pas le succès escompté : malgré

2. On envoya par la suite peintres, photographes et cinéastes, jusqu’à l'" enrôlement des lettrés"(bunshi chōyō 文士徵用) décidé au début de la guerre du Pacifique, qui concerna plus de soixante-dix écrivains (Kitagawa 2003 : 51). 
leur présence effective sur le front, ces auteurs ne parvinrent pas à se libérer du soupçon d'une incapacité essentielle de l'écrivain à accéder à la réalité de l'expérience des soldats.

Jusqu'en 1945, la présence de Hino sur le front et son identité de soldat furent systématiquement soulignées et mises en opposition aux autres textes de littérature de guerre ; l'image du "simple soldat " fonctionnait dans une certaine mesure. Miyoshi Tatsuji 三好達治 qualifie le texte de « compte rendu (issu) d'une pure chronique " (Miyoshi 1938 : 220), Hibino Shirō relève que Mugi to heitai n'aurait jamais pu avoir le succès qu'il a connu si son auteur l'avait visé consciemment, et reprend à son compte le terme de "simple chronique " utilisé par Hino (Hibino 1940 : 80-81). Pour Sugiyama Heisuke 杉山平助, la violence de l'expérience du front ne pouvait pas permettre à Hino de penser la réception de son texte et c'est là encore cette simplicité imposée qui lui a permis de toucher le public (Sugiyama 1939 : 301) : les circonstances commandent un rapport particulier au réel et partant, une écriture particulière. Sugiyama ira jusqu'à affirmer que la description réaliste de la guerre, initiée par Stendhal, est présente naturellement chez Hino (Sugiyama 1939 : 308).

Paradoxalement, la littérarité du texte est d'autre part unanimement constatée, ce qui met la critique devant une impasse : quel statut littéraire attribuer à un texte qui se revendique et qui est lu comme ne cherchant pas à être littéraire ? La question fut déportée sur Hino lui-même : le texte était " naturellement " littéraire parce qu'il avait été écrit par un écrivain, et c'était le caractère de Hino qui touchait le lecteur, parce qu'il informait son écriture. Ce transfert explique l'intérêt présenté par des critiques tels que Furuya Tsunatake 古谷綱竹 pour les textes précédents de Hino, et d'une manière générale pour la psychologie de l'auteur : on s'assurait en même temps de la cohérence du personnage (dont l'" humanisme " semble être le trait principal) et de son authenticité dans la trilogie en les comparant à des textes visiblement plus apprêtés (Furuya 1939: 270). Ce mouvement amena également à aborder le problème de l'impact de la guerre sur Hino, sur lequel le discours de propagande fut particulièrement saillant. La guerre est reconnue tantôt comme le révélateur, tantôt comme l'origine de la nouvelle simplicité de Hino. En rendant l'homme plus simple, elle simplifia également son style, ce qui lui permit de toucher l'ensemble de la population japonaise. Itō Sei 伊藤整 vit quant à lui dans la littérature de guerre - dont il fait de Hino le plus grand 
représentant - le modèle d'une nouvelle littérature japonaise, plus ancrée dans le réel et qui réunit auteurs et lecteurs (Itō 1944: 6).

Avec la publication de Mugi to heitai, le nom de Hino Ashihei devint ce que Michel Foucault désigna sous le terme de "fonction-auteur " quand il tâcha d'aborder la question du mode de réception des textes littéraires : le nom d'auteur indique "qu'il s'agit d'une parole qui doit être reçue sur un certain mode et qui doit, dans une culture donnée, recevoir un certain statut " (Foucault 2001 : 826). En l'occurrence, le lecteur contemporain était en position d'attendre une parole authentique de soldat, un texte véridique fondé sur une expérience vécue, un récit qui lui permette d'appréhender la réalité de la guerre. Fonction-auteur et écriture sont cependant dans une relation d'interdépendance puisqu'il faut également considérer que ces textes eux-mêmes participent à la construction de ce statut; cela est d'autant plus vrai dans le cas de Hino qui, bien que lauréat du prix Akutagawa, demeurait en 1937 un auteur relativement confidentiel. C'est à cet aspect de la question que nous voudrions ici nous intéresser : nous nous proposons au cours de cette étude de montrer comment le texte amène son lecteur non seulement à accepter qu'il est bien face à un authentique récit de soldat, mais aussi à considérer que ce récit vaut pour tous les soldats ; c'est en affirmant une écriture ultra-personnelle fondée sur l'expérience singulière de l'auteur que Hino parvient à remplir ce qui pourrait être défini comme le contrat de lecture du récit de guerre, qui doit dire quelque chose de la guerre dans ce qu'elle a de commun à tous ceux qui la vivent. En somme, Mugi to heitai définit sa propre valeur par le biais de procédés proprement littéraires dont le plus évident est, comme nous le verrons, le fait de se revendiquer comme un texte non-littéraire, rejouant ainsi au sein du texte la doxa selon laquelle la littérature ne peut être authentique.

La mise au jour de ce mécanisme se fera d'abord au travers d'une analyse de la mise en scène de l'authenticité du texte : le dispositif paratextuel de Mugi to heitai qui vise à brouiller les frontières entre texte et réalité s'accompagne d'une rhétorique du récit personnel, défini par opposition à une littérature apprêtée dont l'image est elle-même construite au sein du texte. Nous nous attacherons par la suite à montrer que l'authenticité est une condition nécessaire, mais non suffisante du récit de guerre, puisqu'il importe également de définir dans quelle mesure cette parole individuelle peut valoir pour celle des autres soldats; ce glissement est rendu possible d'une part par un brouillage des focalisations qui permet à Hino de parler 
à la place des soldats, et d'autre part par le jeu de miroirs mis en place de fait quand le narrateur intègre à son texte la parole des soldats.

\section{La mise en scène d'une écriture authentique}

Ils ne font pas de rhétorique, ils livrent à nu les expériences intérieures comme extérieures, de manière véridique et directe, chaque page est payée de sang. Plus ils sont capables de sobriété d'expression, plus ils nous saisissent. (Philipp Witkop, cité par Beaupré 2006 : 69)

Ces lignes datent de 1917 : elles sont écrites par le professeur Philipp Witkop (1880-1942, l'un des spécialistes de la littérature de guerre de l'époque) pour décrire la production des " écrivains combattants » de la Première Guerre mondiale, mais il est frappant de constater que le texte de Hino fut lu d'une manière tout à faire similaire. La comparaison a cependant quelque chose de trompeur : elle dessine l'idée d'une universalité de l'écriture de guerre, qui s'imposerait uniformément à tous les écrivains, et devrait logiquement aboutir à l'uniformité de leurs textes; or l'expérience de la guerre est dans ce type de textes une expérience individuelle, c'est là ce qui fonde leur autorité, et c'est ce que l'écriture a à charge de rendre. L'étonnante portée des propos de Witkop nous paraît plutôt être le signe d'attentes de lecture qui sont, elles, toujours les mêmes, et que l'on retrouvait déjà dans le proverbe latin inter arma silent musae; nul doute qu'elles s'appliquaient également aux textes de Hino, alors même qu'il était le premier soldat-écrivain japonais.

L'édition originale de Mugi to heitai était très fortement marquée par la volonté de présenter un texte aussi crédible que possible. Son appareil paratextuel comprend la postface du major Takahashi, le supérieur hiérarchique de Hino au sein du service d'Information, plusieurs photographies et une seconde postface de Umemoto Takemata 梅本竹馬太 ${ }^{3}$ (tous deux sont des

3. Umemoto Takemata (ou Umemoto Samaji 梅本左馬次) (1906-1973) ouvrit au début des années trente un magasin de photographie qui servait également de quartier général au groupement marxiste révolutionnaire dont il était membre, avant de commencer à travailler comme photographe indépendant. Il fut arrêté en 1934 et emprisonné pour deux années, à la suite desquelles il déclara renoncer à ses opinions politiques (c'est ce que l'on appelle le tenkō 転向, littéralement " retournement ") et entra à la Dōmei tsūshin sha 同盟通信社 (agence de presse nationale), puis fut rattaché en 1938 
personnages récurrents du texte), une carte de la région de Xuzhou et une préface rédigée par Hino. Les photographies (qui représentent dans leur grande majorité des soldats en marche ou au repos, parfois des civils, en tout cas jamais de combats ni de paysages) sont souvent l'illustration exacte de ce qui est décrit dans le texte : elles constituent une caution " objective» (car issue d'un procédé mécanique de reproduction du réel) dont le but est clairement de renforcer cet effet. Les postfaces de Takahashi et Umemoto, quant à elles, brouillent la frontière entre texte et hors-texte en leur donnant chair, par leurs signatures et leur prise de parole indépendante de l'auteur : ils ne sont pas des personnages de papier soumis au bon-vouloir de Hino mais des personnes réelles, qui attestent de la réalité que celui-ci décrit. Ainsi, en établissant une équivalence entre les procédés mécaniques de la photographie et le contenu du texte, et en garantissait la crédibilité du texte par deux des personnages qui y apparaissent, cette première édition établissait un rapport de stricte égalité entre texte et réalité.

Les publicitaires reprirent les mêmes arguments : Mugi to heitai est défini dans une publicité parue le mois de sa publication, dans le numéro d'octobre 1938 de la revue Bungei 文藝, comme « un document de sang et de fumée né au cœur des combats ». On y retrouve le texte de la postface du major Takahashi, qui abonde en ce sens :

Le bataillon OO, qui était la clé de voûte de l'avancée des troupes vers le Nord, a réalisé un exploit dans la bataille de Xuzhou. En tant que membre du service d'Information, je l'ai moi-même suivi en compagnie du caporal-chef Tamai, du membre de l'équipe photographique Umemoto, et du chauffeur Nishi. Ce caporal-chef d'infanterie Tamai Katsunori est Hino Ashihei.

J'ai lu le manuscrit de ce carnet de campagne, c'est un journal tout à fait calme et détaché, sans emphase, vraiment ordinaire, mais il est impossible que la sincérité de son contenu et le caractère de Tamai qui s'y révèle ne séduisent pas son lecteur. Tamai a échappé à la mort à Sunwei ${ }^{4}$. (Hino $1938: 231$ )

au service d'Information où il travailla avec Hino. Ces informations sont extraites du site de l'association Sukarabe スカラベ会, qui constitue depuis 1998 une base de données des activités culturelles et littéraires du sud du Japon ; on y trouve notamment un dictionnaire biographique particulièrement riche : http://www.k3.dion.ne.jp/-scarabee/ sukarabe-jinmei.htm

4. Toutes les traductions sont de l'auteur. 
Dans un registre semblable, la préface signée par Hino a l'avantage de poser explicitement certains éléments présents de façon diffuse au sein du texte, aussi souhaiterions-nous en citer plusieurs passages :

Mugi to heitai est le journal que j'ai tenu quand, en tant que soldat rattaché au service d'Information, j'ai suivi l'armée dans la campagne de Xuzhou, dont on dit qu'elle fut une victoire historique. [...] Je suis au cœur des champs de bataille, continuellement exposé à des épreuves inexprimables, je ne comprends rien et suis comme aveuglé parmi les pensées sur cette grande guerre; quand bien même viendrait le moment où je pourrais aborder cela sous forme de littérature, quand dans un lointain futur j'aurai quitté le champ de bataille pour fouler à nouveau le sol du pays natal, il me faudra revenir tranquillement sur cette expérience et y mettre de l'ordre, car pour l'instant je n'ai pas les mots qui conviennent à cette grande réalité. Je crois que trouver les mots pour cette vérité qu'il faut dire sur la guerre est une entreprise de valeur qui sera le travail de ma vie, mais je me disais que, pour toutes sortes de raisons, je ne voulais pas parler de la guerre pour le moment. Cependant, en un autre sens, j'ai pensé que par les temps qui courent, même la chronique de l'expérience directe d'un soldat qui se trouve au cœur des champs de bataille pourrait avoir une utilité, aussi ai-je fait le choix de noter les choses telles quelles. [...] Ce texte n'a aucun rapport avec la situation ou la stratégie du front de Xuzhou, je me suis simplement contenté d'organiser et de mettre au propre le journal que j'ai écrit chaque jour en accompagnant l'armée. Il ne s'agit évidemment pas d'un roman.

À la suite de l'incident, on publia des ouvrages sur la guerre comme s'il en pleuvait. Nombre de brillantes personnes vinrent sur le champ de bataille, et écrivirent nombre de brillants ouvrages. Rédigés dans une langue émouvante et prenant place sur le champ de bataille, d'exaltants récits d'exploits héroïques, des anecdotes édifiantes qui feraient sangloter de courageux démons, des histoires passionnantes, des romans à l'intrigue pleine d'audace furent écrits les uns après les autres, et paraissent aujourd'hui encore sans discontinuer. Chacun de ces textes était important, tous étaient formidables. Que parmi eux se trouve mon ennuyeux carnet de campagne, modeste et plat, écrit dans une langue monotone, me fait rougir de honte. (Hino 1938 :3)

Hino ne nie pas sa qualité d'écrivain (présenter son expérience "sous forme de littérature " sera le "travail de [sa] vie ") mais présente Mugi to heitai comme le fruit d'une expérience où la littérature, en tant que (ré)organisation rétrospective du réel, n'a pas encore sa place; le texte est un texte "pré-littéraire " au sens où il est écrit immédiatement par celui qui fait l'expérience de la guerre, il est un échec littéraire (les mots ne sont pas "adaptés ") car son auteur n'est justement au moment de l'écriture qu'un soldat " au cour des champs de bataille " qui n'entend rien à " la situation ou [à] la stratégie du front ». Par ailleurs, l'évocation de la 
littérature de guerre des autres écrivains est clairement placée sous le signe de la défiance : la guerre de Hino (c'est-à-dire la guerre authentique) n'est ni romanesque ni exaltante mais " ennuyeuse ", elle n'est pas l'affaire de héros mais de simples soldats dont il fait partie.

Dans Poétique du récit de guerre, Jean Kaempfer isole trois traits distinctifs du récit de guerre moderne (1998: 7-12). D'abord, la " perspective d'un personnage dépassé par les événements ", c'est-à-dire le fait que la guerre soit vue à hauteur d'homme ; les personnages sont jetés dans des conflits qui les dépassent, et surtout dépassent leur entendement : les relations de cause à effet n'existent plus, on se contente d'enregistrer des sensations, des phénomènes subis. La technique, qui a sophistiqué à outrance les moyens qu'ont les hommes pour s'entre-tuer, a créé des guerres qui ne sont plus des affaires d'hommes, et c'est par un retour à l'échelle de l'individu que l'écriture moderne de la guerre illustre cette impossibilité de comprendre la violence des combats. Ensuite, " un univers déshumanisé ", c'est-à-dire la perte complète de repères qu'induit la violence environnante. Enfin, la dénonciation du " mensonge épique " qui vise par contraste à rendre crédible ce nouveau type d'écriture. Malgré son appartenance à un appareil de propagande dont on pourrait attendre qu'il lisse les représentations, il est clair que Mugi to heitai s'inscrit dans ce mouvement. En l'état actuel des recherches (la critique japonaise prenant principalement la forme de monographies), il est difficile d'évaluer précisément la place qu'eut ce type de discours au sein de l'ensemble des représentations de la guerre, mais le succès massif de Mugi to heitai permet a minima d'affirmer que ce phénomène ne fut pas marginal.

La valeur que Hino attribue à son propre texte est soutenue par le choix même du genre du journal, qui inscrit Mugi to heitai au sein du vaste et informe corpus des authentiques journaux que les soldats tenaient durant la guerre ; même si ces textes étaient effectivement cantonnés à une écriture pour soi ou pour des proches, leur existence était connue de tous et faisait régulièrement l'objet d'une mise en scène complexe lorsque certaines revues choisissaient d'en publier des extraits. Mugi to heitai se trouve ainsi aux prises avec un intertexte en grande partie imaginaire qui met d'emblée le lecteur face à un récit qui revendique son authenticité. En voici les premières lignes : 
4 Mai

Il fait beau, le ciel est clair.

Je m'arme pour le départ et vais dans la chambre du lieutenant-colonel Mabuchi. Quand j'entre, il m'écrit une lettre à l'intention du major Takahashi ainsi que mon ordre de mission, et m'indique en détail la situation du service d'Information de Bengbu et les sections du service qui sont au front, puis il demande à mademoiselle Tsuji, qui s'occupe du service, de lui apporter de la bière, me dit « vous en prendrez bien une ", l'ouvre puis la verse. (Hino 1938 : 9)

Le journal s'ouvre sur une date, sans mention de l'année, suivie immédiatement de considérations météorologiques : un dispositif aussi typique (on est face au lieu commun par excellence du journal) pourrait paraître caricatural, mais il permet de mettre au jour plusieurs caractéristiques de l'ensemble du texte. Pour Roland Barthes, "le temps qu'il fait » est caractéristique du journal parce qu'il permet d'écrire même lorsqu'il ne se passe rien : " ... n'ayant pour dessein que de dire le rien de ma vie [...] le journal use de ce corps spécial dont le "sujet" n'est que le contact de mon corps et de son enveloppe et qu'on appelle le temps qu'il fait. " (Barthes 1953 : 167). Il y a en effet un aspect déceptif, qui fait partie des effets de réel du journal, dans cet incipit : comment un auteur qui vit quotidiennement la plus grande guerre que le Japon ait connue pourrait-il dire qu'il ne se passe rien ? Néanmoins, cette attention au " contact $[\mathrm{du}]$ corps et de son enveloppe " fait sens en soi : ce n'est pas un positionnement d'écrivain, mais d' "individu moyen ", elle place (d'autant plus qu'elle est inaugurale) le narrateur sous le signe d'une banalité du rapport au réel. Ce « rien » a de l'importance pour Hino pour la même raison qu'il en a pour n'importe qui, parce qu'il est là, simplement.

Ce «temps qu'il fait » se substitue d'autre part à la justification de l'écriture, courante en début de journal : rien ne permet explicitement au lecteur de saisir ce qu'est ce texte auquel il est confronté. Sa fonction reste obscure : portrait de soi, recueil d'une expérience dont Hino voudrait garder une trace, juxtaposition gratuite d'observations? Avant l'écriture, il y a nécessairement eu une décision d'écrire qui échappe au lecteur puisque le texte est le résultat de cette décision, et qui le laisse face à un vide inaugural. Ce vide, anti-didactique, met d'emblée le lecteur dans une position de voyeur qui prend ce fragment de vie en cours de route, sans y avoir été invité. En ce sens, le lecteur semble n'avoir pas d'autre place dans le texte que celle qu'il croit conquérir : déchiffreur d'un sens qui ne lui est pas destiné, chercheur 
d'une intention qui lui a été cachée. L'effet de réel fonctionne ainsi complètement, le journal est un objet radicalement étranger comme le serait tout journal inconnu. Le récit est un récit de l'être-là, qui suit les mouvements de la conscience du narrateur selon qu'elle se tourne vers tel ou tel objet, prend de fait la forme d'une juxtaposition d'observations, tout en niant la possibilité d'une encyclopédie partagée avec le lecteur (c'est-à-dire donc en niant le lecteur) par l'usage de noms propres totalement obscurs : on passe de "la situation du service d'Information de Bengbu " à " mademoiselle Tsuji " sans autre transition que la succession chronologique des événements.

Le lecteur est d'emblée mis face à un texte qui ne s'adresse ostensiblement pas à lui, ce qui semble répondre à une distinction manichéenne des fonctions respectives de l'écriture d'écrivain (le pour autrui) et de soldat (le soldat n'a pas de public). Ce dispositif textuel joue sur une certaine doxa, un ensemble d'a priori idéologiques et littéraires plus ou moins explicites au sein du texte : l'idée que l'écriture personnelle se fonde sur une sincérité dont la fiction est par essence incapable, l'idée que la conscience chez l'auteur de son public exclut de fait une telle sincérité, l'idée que toute littérature est une représentation biaisée (du fait même qu'elle est représentation) de la réalité, l'idée qu'il peut exister un texte qui ne soit pas représentatif mais simplement présentatif, constituent un réseau de préjugés qu'active Mugi to heitai. Celui-ci est particulièrement explicite dans un passage où Hino écrit puis refuse d'envoyer un vers à un ami :

À Kankichi, j’ai écrit ce vers :

Comme une étincelle sur le chemin d'un kappa prêt à enterrer sa vie

Mais quand auparavant, la veille du débarquement dans la baie de Hangzhou, les lettres jusque-là interdites avaient été autorisées, j’avais simplement écrit : "Enfin, je débarque au nord de la baie de Hangzhou. » J'avais été incapable d'écrire que les balles des Chinois ne pouvaient pas m’atteindre, ce tragique sincère était très différent de mon état d'esprit actuel, alors que je pars vers le front. Je pourrais mourir dans cette campagne, mais il m'a semblé y avoir quelque chose d'apprêté dans les mots que je venais d'écrire, et je n'ai pas envoyé le vers à Kankichi, je l'ai recopié à la première page de mon carnet. J'ai pensé que si personne ne le voyait après ma mort, cet apprêt n'aurait pas d'importance. (Hino $1938: 32$ )

Ryū Kankichi 劉寒吉 (1906-1986, de son vrai nom Hamada Rikuichi 濱田 陸一) est un ami de Hino, romancier et poète, avec lequel il avait 
notamment participé à la revue littéraire Toranshitto とらんしつと au milieu des années 1930, mais celui-ci est ici simplement nommé, sans être présenté. Le refus du poème illustre la disqualification du langage poétique en raison de son "apprêt " : on y lit d'une part l'existence assumée d'une tentation proprement littéraire, d'une recherche du mot juste inaccessible au langage commun, et d'autre part un soupçon qui pèse sur l'idée même d'une expression travaillée en tant que rapport médiat au réel - qui par contraste renvoie du texte l'image d'une immédiateté rendue possible par une langue sans apprêt.

Hino choisit cependant de conserver ce vers pour lui, signe que ce n'est pas tant l'apprêt poétique en soi que le regard porté par autrui sur cet apprêt qui le gêne. Le problème semble plutôt résider dans le fait d'écrire pour autrui, ou pour être plus exact dans le fait d'être lu par autrui, comme si la poésie devait être cantonnée à une communication de soi à soi, dans l'intimité la plus absolue - ce qui n'est, en l'occurrence, possible que dans le journal. Il faut cependant noter que Hino ne pousse pas la mise en scène jusqu'au bout : le vers censé être à la première page du carnet ne figure pas dans l'édition originale de Mugi to heitai, ce qui instaure de fait une distance entre le carnet figuré et le livre que le lecteur a sous les yeux, et rompt en quelque sorte le charme du journal.

Le sur-investissement du genre du journal, caractéristique de l'ensemble de Mugi to heitai, semble opposer l'écriture de soi à d'hypothétiques codes classiques du récit de guerre, mais il s'agit plutôt de construire implicitement, justement parce que l'écriture surjoue sa banalité, sa monotonie, sa fadeur, l'image du récit de guerre que ce texte n'est pas. Ce faisant, l'écriture de soi construit de la même manière l'image du lecteur de ce récit de guerre hypothétique que le texte est donc voué à décevoir, et impose un mode de lecture semblable à celui de n'importe quel journal. Dans une perspective similaire, Mugi to heitai semble également nier la possibilité d'un projet narratif : l'expérience de la guerre est constituée d'accidents imprévisibles qui refusent de se laisser appréhender dans une perspective globalisante, elle est une succession d'événements dont le soldat ne peut comprendre l'enchaînement car ils dépassent l'individu (le « je ») et le temps humain (la journée). Pour reprendre les termes de Michel Braud, le journal est (au mieux ?) " un projet d'écriture largement indéfini " (Braud 2006 : 269). Absence de projet narratif ne signifie pas pour autant absence totale de structure : le texte décrit l'expérience de Hino durant la campagne de 
Xuzhou et donc un voyage dont le but est l'arrivée dans cette ville. Au fur et à mesure des jours, Hino se rapproche de la zone des combats, puis avance avec elle, et est ainsi de plus en plus amené à vivre et à décrire des scènes de combat. Cette gradation est censée n'être que l'effet du statut de témoin de Hino ; dans la mise en scène de l'écriture, cette réalité préexiste au texte et ne peut qu'échapper, en tant que tout, à la conscience d'un auteur qui écrit au jour le jour. Ce retour d'une structure, ou plutôt d'une intrigue a priori évacuée en raison de l'authenticité (de la non-littérarité) du texte s'opère malgré l'auteur, comme si l'histoire (l'Histoire ?) s'imposait d'elle-même : le réel a un sens, une direction, un "projet " qui ne dépend pas de Hino. À ce titre, il est placé en tant qu'auteur dans une situation d'échec, puisqu'il est incapable d'agir sur le réel ou même de l'organiser dans l'écriture ; se bornant à un rôle de témoin " myope ", il semble laisser au réel le soin de s'organiser lui-même.

On peut cependant pousser l'analyse plus loin : s'il existe bien un projet qui structure le réel, observable à travers le retour d'une intrigue au sein du journal, celui-ci n'est pas apparu ex nibilo. Il est l'effet de la volonté de l'armée, sans qui il ne pourrait y avoir de campagne, ni a fortiori de récit de campagne ; cette armée comme principe d'organisation du réel dépasse tous les individus - tous les soldats sont ici égaux, et Hino ne prétend pas à ce titre être différent. Feignant de s'en remettre naïvement à la réalité quotidienne, il cède en fait son auctorialité à l'armée à qui revient la charge de donner un sens au réel : l'écrivain se laisse porter par une guerre qui lui échappe, il épouse le projet de l'armée en l'accompagnant. En outre, si l'on accepte de considérer que l'armée " écrit " une intrigue que Hino ne fait que reproduire, ne peut-on pas voir là une image de l'ensemble des textes littéraires produits à l'époque dans un but plus ou moins avoué de propagande, en tant qu'ils sont une forme d'écriture où l'auteur se soumet à une entité qui lui " dicte » ses textes ? Dans de telles conditions, l'écrivain cédait de fait - à un degré qui reste aujourd'hui encore à définir - son auctorialité à l'armée, puisqu'il n'était plus le seul à décider de son texte.

L'authenticité revendiquée du journal n'est cependant pas le seul critère qui différencie Mugi to heitai, dans le système de valeurs mis en place au sein du texte, de l'image de la littérature que l'auteur dessine en creux. Il importe également que le texte soit capable de dire le réel, et cette foi affichée dans la capacité du langage à présenter (plutôt que représenter) le monde trouve son 
expression la plus claire dans l'utilisation d'une mimesis poussée à l'extrême, à travers laquelle le texte devient proprement un double du réel :

\begin{abstract}
À l'intendance, on nous a distribué diverses provisions. On nous a déclaré qu'il fallait se résigner à ce que désormais les vivres ne nous parviennent plus normalement. Recevoir du riz japonais m’a rendu plus heureux que jamais. J'ai reçu de la marinade de daurade au miso, des radis, des boîtes de bonite, de la soupe miso en poudre... Ce qu'on appelle "soupe miso en poudre » se trouve dans de petits sachets, il est écrit sur le mode d'emploi : " Pour faire de la soupe miso, diluer un sachet dans le double de son volume d'eau (remplir jusqu'au couvercle de la gamelle) ou ajouter un volume d'eau équivalent et faire bouillir. " Apparemment il y a aussi du fu et des algues, c'était une première, j'ai apprécié que les soldats puissent malgré tout boire de la soupe japonaise. (Hino $1938: 74$ )
\end{abstract}

Ce passage tient d'un procédé quasi-photographique par lequel Hino se contente de reproduire un texte dont il est lui-même le lecteur. En en présentant la forme brute, l'auteur invite à y poser le même regard que lui, et recrée ainsi ce regard chez le lecteur ; tous deux sont égaux devant ces mots, Hino (sup)pose, en choisissant de les présenter tels quels, qu'il sera possible de les lire de la même façon que lui. Le cas le plus fréquent de ce type d'utilisation de l'écriture se trouve dans les formules composées de quatre caractères que Hino trouve accrochées aux maisons chinoises, et qu'il reproduit frénétiquement sans glose ni explication. Le rapport de Hino au réel se révèle alors égal au rapport du lecteur à la reproduction du réel, puisque ce type de mimesis permet, au moyen d'une pseudo-neutralité, d'en assurer l'intelligibilité (ou du moins de rendre ce réel aussi intelligible au lecteur qu'il ne l'est à luimême) ; par ce type de processus, le lecteur peut ainsi lire le texte sans mettre en péril la mise en scène de l'écriture personnelle puisque la mimesis est un pour soi qui ne se trouve qu'accidentellement faire sens pour autrui.

Dans une certaine mesure, il est en fait possible d'étendre ce type de réflexion à l'usage de la langue japonaise dans l'ensemble du texte : l'écriture se veut la plupart du temps aussi neutre que possible, se bornant à enregistrer des phénomènes (extérieurs comme intérieurs) observés par Hino ; la langue met en scène une relation d'égalité entre les mots et ce qu'ils désignent. La distance au réel qu'implique nécessairement l'écriture, le processus de conceptualisation consubstantiel à l'usage de la langue, le travail intellectuel que requiert tout texte de son auteur se trouvent éclipsés par l'apparence d'un style purement neutre. 


\section{Figures du porte-parole}

\section{L'instabilité des focalisations}

Dans l'un des passages les plus déroutants de ce texte à la mise en scène faussement simpliste, Hino aborde explicitement la question de la valeur de son expérience de guerre. Cet extrait fait suite au récit des quarante-huit heures les plus périlleuses du Mugi to heitai, au cours desquelles les soldats qu'accompagne Hino ont été attaqués par surprise dans la ville de Sunwei 孫圩 qu'ils venaient de conquérir ; après avoir finalement remporté la victoire, les soldats profitent d'un moment de répit :

La scène ressemblait bien à la courte halte qui suit une marche. Les soldats ne paraissaient absolument pas sortir d'un combat à mort pour s'avancer vers un nouveau combat à mort à venir. Cet air indifférent des soldats, dont l'officier d'état-major Nakayama avait un jour dit qu'il imposait le respect, c'était cet air-là. Je me suis rappelé la journée que j'ai passée à Sunwei, et je me suis aperçu que moi non plus, je ne ressentais pas cela comme une expérience particulière. Sur le champ de bataille, il n'y pas d' "expérience particulière ". Ça ne vaut pas spécialement la peine d'en parler. Les mêmes journées se suivent de la même façon. Depuis Shanghai, depuis Nankin, sur la route vers Xuzhou, et plus loin, les champs de bataille se suivent indéfiniment. L'impression que m'a fait Sunwei fait partie du quotidien des soldats. Et déjà, cette impression disparaît. Il ne s'agit pas d'avoir cédé à cette impression. Il ne s'agit pas d'avoir rejeté cette impression. Il s'agit de l'avoir dépassée. Cette situation, que des mots ordinaires tels que " peine " ne peuvent exprimer pleinement, cette situation qui au début écrase les soldats, ils la dépassent dans l'instant. Alors que je me tenais debout, déconcerté, sur la colline des grenadiers, l'ordre du départ a été donné. (Hino 1938 : 200-201)

Rétrospectivement, voir l'auteur de milliers de pages de récits de guerre affirmer qu'il n'y a pas d'" expérience particulière " et que "ça ne vaut pas spécialement la peine d'en parler " peut évidemment sembler d'une ironie consommée, mais même dans le cadre restreint du texte en question, l'affirmation pose évidemment problème. Cette affirmation est strictement incompatible avec l'existence même du texte qui fait l'objet de ce travail, qu'il soit considéré au premier degré comme écrit pour soi, ou au second, en tant que texte publié et imposant ainsi au lecteur l'idée de son intérêt. Le jeu est évidemment trop ostensible pour être pris à la lettre, mais il nous dit quelque chose des contradictions inhérentes au texte : celui-ci ne devrait pas exister si Hino était effectivement un soldat comme les autres, si 
ce journal était un journal comme les autres. En ce sens, Hino détient (ou plutôt se confère) une autorité qui lui est propre et le met dans la position paradoxale d'être celui qui dit qu'il n'y a rien à dire ; il révèle d'autre part à quel point il est lui-même conscient que cette posture du soldat comme les autres prenant la parole au nom de tous est par définition intenable.

La rhétorique du récit personnel, bien qu'elle ne soit pas exempte de contradictions internes, ne doit cependant pas être considérée comme un obstacle à la valeur collective de ce même récit ; au contraire, cette rhétorique contient en soi les conditions de son dépassement et se révèle nécessaire à la figure de porte-parole que le narrateur du récit de guerre doit incarner. Le rapport établi dans le texte entre sa parole et celle des autres soldats permet de dépasser cette aporie du texte que Hino se plaît à dénoncer quand il affirme que "ça ne vaut pas spécialement la peine d'en parler ", en isolant ce que toutes ces voix qu'il choisit de retranscrire ont en commun : la raison pour laquelle elles valent qu'on en parle. L'exemplarité dans laquelle s'inscrit de fait le texte repose sur une forme d'homogénéité de l'expérience de guerre, ou du moins sur l'idée que la réalité à laquelle les soldats font face à quelque chose de semblable pour tous. En abordant Mugi to heitai à la lumière du concept de polyphonie, il est en effet possible de montrer que le récit individuel se dépasse lui-même en mettant en scène une certaine uniformité de l'expérience de la guerre dont nous voudrions tenter de définir les modalités. Ces récits dans le récit, cette polyphonie récurrente constituent un genre de mise en abyme formelle de la situation d'énonciation du texte, puisque Hino devient le destinataire de souvenirs de guerre comme le lecteur reçoit sa parole à lui ; ils sont ainsi la preuve par le fait de la communicabilité de l'expérience de guerre, sur laquelle repose l'écriture de Hino. Leur présence dans un récit personnel de guerre pose cependant problème : sont-ils un moyen de compenser le champ réduit d'une expérience personnelle, limitée par le parti pris d'écriture de Hino, ou au contraire l'affirmation que toutes ces expériences se valent ? La question de la valeur de ces souvenirs de soldats est double : c'est à la fois celle de leur valeur intrinsèque (individuelle ou commune) et celle de la valeur qu'ils donnent aux souvenirs de Hino.

La notion de porte-parole nous permet en effet d'aborder le texte en tant que collection de voix, particulièrement manifestes dans les focalisations instables de certains passages. C'est ce à quoi Hino semble nous 
inviter dans l'extrait suivant, qui prend place au cours de l'attaque de Sunwei évoquée plus haut :

J'ai été entièrement jeté entre la vie et la mort. Je me suis résigné à mourir. Ce que, bizarrement, je trouvais audacieux, vacille maintenant comme un bâtiment sans fondations. J'avais comme l'étrange conviction que les balles ne m'atteindraient pas. Ce n'était qu'un moyen de me rassurer. D'innombrables obus de mortier tombent et explosent autour de moi. À chaque fois, ils font des victimes et montrent la couleur du sang. Je ne dois ma vie qu'au hasard, qui a voulu que ces obus ne tombent pas sur moi. À l'idée que de précieuses vies soient si facilement blessées, une violente colère s'empare de moi. Élever une seule de ces vies jusqu'ici s'est fait au prix de nobles, indescriptibles efforts, payés sans regret. Ces vies portent encore un précieux futur à construire. Et puis, tous les soldats qui sont là sont les fils de leurs parents, les maris des femmes qu'ils ont au pays, les pères des enfants qu'ils ont laissés pour venir, ce sont des êtres humains d'une extrême importance pour notre pays. Les soldats portent leur nostalgie, ils gardent tous dans leur cœur, comme un objet précieux, le rêve de leur retour triomphal. Et un coup de hasard, en un instant, enterre tout cela. Ce ne sont pas des réflexions rétrospectives. C'est une impression banale qu'on a sur le champ de bataille. Il ne s'agit pas de regretter la vie que l'on donne pour le pays. Mais je ne peux pas réprimer cette sensation de colère qui bouillonne en moi. (Hino $1938: 144$ )

Cet extrait, qui est comme un pendant exact à celui cité précédemment, nous permet d'aller encore plus loin dans le paradoxe du récit de guerre de Hino : il n'y a pas d'" expérience particulière " parce qu'il n'y a que des " expériences particulières ». Le mouvement des focalisations observable ici traduit ce paradoxe : la violence impose d'abord à Hino un retour sur luimême et sur sa fragilité, c'est-à-dire sur sa condition d'être humain, puis le ramène par ce dénominateur commun à ses camarades qui sont dans la même situation. Son indignation est à la fois celle d'un individu devant l'injustice qu'il constate, et celle du membre d'un groupe devant l'injustice qu'il subit. La conclusion incarne expressément cette union, scellée par la mort que tous les soldats ont en partage ( Il ne s'agit pas de regretter la vie que l'on donne pour le pays").

L'ambiguïté de sa position se révèle cependant quand il commente son propre texte : "Ce ne sont pas des réflexions rétrospectives. C'est une impression banale qu'on a sur le champ de bataille. » Hino semble ici chercher à se protéger du soupçon de n'être pas conforme à l'esprit attendu, au cliché du soldat simple soumis à une violence qui coupe toute possibilité de réflexion - cliché qu'il construit d'ailleurs lui-même en opposant les termes 
" réflexions » et "impression ». En termes de focalisation, l'omniscience que dénote sa capacité à déchiffrer les signes (un soldat signifie les « nobles, indescriptibles efforts " qui ont construit sa vie) et à parler pour tous est ici explicitement justifiée par le fait que son expérience de la violence est identique à celle des autres ; l'idée qu'il nous fait part d'une "impression banale " semble nous ramener à l'image d'un porte-parole " comme les autres ", puisque tous les soldats savent que tous les soldats sont victimes de l'injustice de la guerre.

Ce que dit Hino du texte est cependant exactement le contraire de ce qu'il fait : ce qu'il écrit ne peut par essence qu'être le fruit de réflexions rétrospectives, et sa volonté de le nier montre paradoxalement à quel point il en est conscient. La recherche d'une voix extérieure à Hino qu'il retranscrirait dans le texte se heurte à l'idée même d' "impression banale » : celle-ci existe hors du langage chez les soldats, elle est une sensation qui n'est formulée par personne d'autre que Hino, et l'est uniquement au moment de l'écriture. Nous sommes donc bien ici face au porte-parole d'une parole qui n'existe pas en tant que telle et à laquelle seul le narrateur, parce qu'il est en vie et parce qu'il est écrivain (ou du moins parce qu'il écrit), peut donner corps. La tonalité romantique qui affleure dans ce rapport à la survie demeure rare au sein du texte - elle trancherait trop avec l'image du simple journal si elle était généralisée - mais n’en demeure pas moins une composante essentielle. C'est un romantisme pour ainsi dire pragmatique, qui dépend non pas d'un regard a priori sur le monde mais bien de l'expérience réelle de la mort ; en ce sens, la prise de parole tient à la fois d'un devoir de témoigner et d'un besoin de laisser une trace. Les circonstances de l'écriture de Mugi to heitai, et en premier lieu le fait que Hino ait été membre d'un service d'Information, participent évidemment - bien qu'à un autre niveau - à ce phénomène. Dans un texte paru peu avant sa mort ${ }^{5}$, il relate par exemple le discours que lui tint son supérieur au service d'Information ${ }^{6}:$ " Il y a bien

5. Il convient cependant de garder à l'esprit que ce texte est suspect à plus d'un titre : il a été écrit une vingtaine d'années après les faits qu'il relate, à une époque où Hino tâchait de modifier son image d'auteur militariste. Pour un traitement plus approfondi de ces questions, se reporter à Rosenfeld (2002) et Ikeda (2000).

6. Il s'agit en l'occurrence de Mabuchi Itsuo 馬淵逸雄, qui fut une " figure clé de la relation entre les opérations de propagande militaire et civile en Chine et au Japon » (Kushner 2006 : 77). 
d'autres soldats qui peuvent combattre en tant que chefs d'escouade, mais il n'y a pas d'autre soldat capable d'écrire et de recevoir le prix Akutagawa. Dans ton cas, servir avec ta plume a dix fois, cent fois plus de sens que travailler une arme à la main » (Hino 1958 : 402).

C'est dans les récits de marche qui jalonnent le texte et donnent indirectement son titre au livre, puisque l'espace qu'il s'agit de traverser est constitué d'incommensurables champs de blés, que l'on trouve les cas les plus marquants et les plus riches de brouillage des focalisations; la longueur et la monotonie de ces marches laissent le soldat face à son corps et semblent conduire à sa dissolution dans celui des autres.

Les nuages de poussière rendent la bouche âpre. La poussière crisse quand elle atteint les dents. On crache de la salive jaune. La sueur coule à flots. Elle imprègne puis traverse l'uniforme. La poussière se colle à la sueur, forme des plaques quand on l'essuie, comme si le maquillage blanc d'un mauvais acteur de théâtre de province s'écaillait. Les soldats marchent sans un mot. Si on leur adresse la parole, ils prennent un air sérieux et ne répondent presque rien. Quand on fait une courte halte, que ce soit dans la poussière ou sur le fumier, ils tombent à la renverse comme si on les y jetait. Ils ont attaché à leur barda des chaussettes qui contiennent des rations de riz pour quelques jours. Leurs sacs sont à coup sûr bourrés à craquer. Ils contiennent aussi des munitions et des grenades. Les soldats étendus, comme s'ils retenaient ce court instant, s'étirent les jambes, relâchent les épaules, font couler de leurs gourdes à leurs bouches, avec précaution, une gorgée d'eau bouillie refroidie. Dans les marches par grande chaleur, l'eau d'une gourde pleine est déjà un réconfort. Il n'y a que des champs de blé à perte de vue, les cours d'eau sont extrêmement rares, et quand bien même il y en aurait, on ne peut pas boire de l'eau trouble. L'eau qu'on a fait bouillir et mise dans la gourde doit être traitée avec précaution toute la journée. Départ. La marche dans la poussière continue. Les sangles du sac à dos mordent l'épaule. Le fusil passe de l'épaule gauche à la droite mais il est hors de question de déposer le sac. La poitrine se serre. On se secoue. Cela va un peu mieux. Les sangles recommencent à mordre l'épaule. Les soldats ont un air indifférent, ils avancent. Ils sont recouverts de poussière, prennent l'apparence de poupées de terre, sont trempés de sueur, continuent à marcher. (Hino 1938 : 60-61)

Le sens exact de cette description est difficile à cerner : le début du texte peut parfaitement être lu comme se rapportant à Hino uniquement, et même les mentions explicites des soldats ne lèvent pas l'ambiguïté puisqu'il en est lui-même un. L'absence de pronoms laisse cette parole dériver de soi à tous et de tous à soi, l'usage de l'atemporel laisse planer le doute sur 
ce qu'il s'agit de décrire : il y a simultanément une peinture universelle de la marche de soldat, et une description du corps de Hino à un moment précis, les deux interprétations présentant l'originalité de ne pas être, au moins linguistiquement, mutuellement exclusives. Tenter d'y voir un portrait de soldats fait par un observateur extérieur met immédiatement à jour la question de la focalisation, incompatible avec une telle interprétation, mais il n'y a d'autre part aucun « je » ou "nous » qui permette de rattacher explicitement le passage à l'expérience particulière de Hino (cet effet est particulièrement subtil dans le texte original, puisque la langue japonaise tolère l'absence de sujet). Le narrateur se fait ici omniscient, il modalise là sa description comme s'il y avait la possibilité d'un doute, il est à la fois l'observateur, l'observé exclusif, et l'observé parmi tous. Le corps particulier, qui est le seul que Hino est censé pouvoir observer avec le degré de précision de cet extrait, se dissout dans sa description sans aucune solution de continuité, comme s'il n'y avait aucune frontière entre lui et le corps commun. Ce texte ne peut ainsi figurer dans Mugi to heitai sans apparaître comme une rupture du contrat de lecture que parce que l'expérience vécue de Hino est commune à tous. La seule façon de maintenir une cohérence dans la narration - nécessaire puisque nous ne sommes pas censés être en présence d'une fiction - est alors soit de se figurer une conscience commune des soldats, dont Hino ferait partie et qui lui permettrait de faire le récit au même titre que n'importe quel autre soldat, soit au contraire de supposer chez lui une capacité exceptionnelle à inférer, de sa propre expérience, l'expérience des autres : on retrouve là toute l'ambiguïté de la posture de porte-parole, à la fois un parmi les autres et plus que les autres. Cette complexité, qui est ici particulièrement saillante, conduit de fait à reconsidérer l'image de Hino comme simple soldat : la communauté de l'expérience, qui tend effectivement à présenter Hino comme un soldat parmi d'autres, trouve ici une expression d'une virtuosité littéraire qui illustre la distance de soi à soi à l'œuvre dans Mugi to heitai : le texte est mené par un narrateur exceptionnel qui semble parfois se plaire à nous le prouver.

\section{La mise en scène des propos rapportés}

Le concept de porte-parole permet ainsi de réinterpréter ces focalisations contradictoires comme les traces d'une polyphonie recréée par Hino à partir d'un " discours muet " qu'il aurait à charge de formuler dans son 
texte et donne l'image, sinon d'une uniformité, du moins d'un continuum de l'expérience de guerre. Hino n'est cependant pas le seul à verbaliser ce discours, qui n'est pas toujours muet : on trouve çà et là, quoique rarement, des passages où Hino rapporte les propos d'autres soldats. Mugi to heitai devient ainsi le lieu d'une polyphonie explicite, qui permet d'aborder sous un nouvel angle la question de la valeur du récit : il devient possible d'interroger l'uniformité de l'expérience de la guerre.

Aucun des souvenirs de soldats rapportés à Hino ne renvoie à des événements qu'il aurait vécus lui-même : on est toujours a minima dans une complémentarité des points de vue (ceci est particulièrement net à propos de l'épisode de Sunwei, dont Hino se fait raconter les événements par ses camarades du service d'Information qui le quittent dès le début de l'attaque) qui semble indiquer une volonté de combler des lacunes de son récit. Pourtant, une part importante de ces souvenirs relève davantage de la pure anecdote :

Umemoto m'a raconté qu'il avait rencontré des soldats en déroute. La nuit était tombée, il régnait une obscurité à ne pas voir le bout de son nez, pendant la marche, Umemoto a entendu des coups de feu dans les ténèbres tout près de lui, il s'est étonné et a demandé ce qu'il se passait, on lui a répondu qu'on avait tiré sur des soldats ennemis en déroute, un soldat qui marchait en tirant un cheval s'était mis à parler au soldat qui marchait à côté de lui, il devait marcher jusqu'alors en silence à cause de la fatigue, mais il s'était rappelé quelque chose et avait eu envie de parler, ce à quoi l'autre n’avait pas répondu, puis il lui avait à nouveau adressé la parole, et il lui avait répondu quelque chose, mais c'était du chinois, il avait trouvé ça bizarre alors il l'avait saisi, puis s'était rendu compte que c'était un soldat chinois, le soldat japonais stupéfait avait crié : Des soldats en déroute se sont mêlés à nous ! et alors ailleurs aussi des gens avaient tenté de s'enfuir de la colonne, il y en avait cinq ou six, [...] ils n'avaient pas pu savoir à cause de l'obscurité, et avaient pensé que c'étaient leurs camarades qui battaient en retraite, les soldats en déroute, heureux, avaient rejoint la colonne en marche, les soldats fatigués ne parlent jamais, quand ils avaient entendu parler, ils n'avaient peut-être pas compris que c'était l'armée japonaise, à Xuzhou il y a des soldats de toute la Chine, ça arrive souvent que des camarades qui viennent de confins opposés ne puissent pas du tout se comprendre, quand des soldats japonais leur avaient parlé, ils avaient certainement pensé que c'étaient des frères d'arme qui venaient d'une région lointaine [...]. Telle était l'histoire d'Umemoto. (Hino 1938 : 220-222)

Cette anecdote qui pourrait paraitre porteuse d'une leçon (la capacité de la langue japonaise à distinguer les amis des ennemis, l'incapacité des Chinois à se reconnaittre entre eux), est surtout centrée sur le ridicule de 
la scène décrite, où alliés et ennemis ne se reconnaissent pas et marchent ensemble en raison d'un quiproquo bouffon, qui frappe. Cette impression est renforcée par le style dans lequel Hino reproduit le discours de Umemoto : une succession de phrases courtes, vivantes et désorganisées, à travers lesquelles il cède littéralement la parole à son interlocuteur, fait de sa propre parole la scène sur laquelle un acteur comique raconte une histoire en feignant d'ignorer, dans un ersatz d'indignation et de justification, qu'il est lui aussi l'un des protagonistes de la farce.

On est là dans un rire de soi qui, s'il était assumé en son nom propre, trancherait très nettement avec le sérieux de la figure du narrateur, de son rapport à la guerre et à son récit (il suffit de se rapporter au passage cité plus haut dans lequel Hino raconte son expérience de l'attaque de Sunwei pour s'en apercevoir). Le mélange des genres que cette anecdote apporte au texte figure la diversité des expériences de guerre, la variété des individus qui la vivent, l'hétérogénéité de leurs voix - une incapacité du narrateur à tout vivre et à tout dire, mais cela n’a jamais été censé être son ambition. Il n'y a rien d'explicite qui justifie l'irruption de cette scène que Hino ne commente pas dans le texte, pas plus que ce souvenir n'est motivé dans le cours d'une conversation : il semble répondre à un besoin spontané de s'exprimer, de partager une expérience vécue avec un interlocuteur capable de la comprendre. Ce n'est finalement qu'accidentellement que Hino semble être le destinataire de ces souvenirs de soldats, exactement comme si l'on rejouait ici la situation d'énonciation du texte : quelque chose de soi est dit, dans un geste de confiance en la capacité de la langue à transmettre un vécu personnel, laissant le destinataire décider de l'intérêt de cette parole. De la même façon que le journal, en tant qu'il est donné à lire, impose au lecteur l'idée qu'il a un intérêt, la verbalisation de ces souvenirs est en soi un indice de leur valeur.

Dans cette perspective, le dernier extrait de Mugi to heitai que nous voudrions maintenant citer est sans doute le plus instructif : Hino y présente à la fois l'histoire qu'on lui raconte et la façon dont lui-même la reçoit. En rejouant dans la diégèse la réception du texte et son effet sur le destinataire, il semble nous entraîner vers ce que pourrait être le sens de son activité d'écriture :

Dans un petit village, quarante ou cinquante soldats se reposaient. C'était le premier bataillon d'infanterie que je voyais. Ils étaient trempés de sueur et portaient 
des uniformes maculés de terre rouge, assis chacun à sa guise à l'ombre des maisons en terre ou sur les talus. Deux ânes aux oreilles démesurément longues, chargés de bagages, étaient attachés à un saule. Il y avait un certain adjudant Hirose, et j'ai entendu l'histoire de la bataille de Zhangbaying, son premier combat sanglant depuis qu'il avait traversé la Huaihe. C'était une bataille extraordinairement difficile, le 6 au matin, le bataillon Hitomi qui avait lancé l'attaque déplorait plus d'une centaine de morts et des centaines de blessés. Le soir venu, à cause de la pluie dans les tranchées, la terre rouge s'était changée en un bourbier dans lequel ils avaient avancé comme s'ils nageaient, les mitrailleuses et les armes de poing plongées dans la boue étaient devenues inutiles, et ça avait été un combat au coude à coude comme si, levant les yeux lorsqu'ils rampaient dans la boue, ils s'étaient trouvés face à l'ennemi, il s'ensuivit un violent corps à corps. C'était une histoire à faire frémir. Je me dis que la terre rouge collée aux uniformes des soldats remontait à ce moment-là, et alors que je l'observais à nouveau, comme on regarde quelque chose de noble, l'adjudant Hirose poursuivit le récit de la dure bataille : Il paraît que le commandant Fujino est parti à l'assaut avec dix-sept soldats, et même une fois la position prise, l'ennemi a contre-attaqué deux fois, nous les avons bien sûr repoussés mais malheureusement, finalement, dans ma section aussi il y eut cinq morts et seize blessés, dit-il tristement. (Hino 1938 : 48-49)

L'omniprésence de la boue, l'insistance sur la difficulté des combats, la réalité de la mort des camarades sont autant de signes d'une mémoire cliché du combat qui est en train de se former à la fois entre les personnages dont Hino fait partie et hors du texte, dans le réseau des représentations réalistes de la guerre véhiculées par les différents médias. D’autre part, ce récit passe alternativement de l'individuel au collectif sur un mode semblable à celui que Hino lui-même utilise pour raconter ses batailles, et Hino répercute cette double valeur sur sa façon d'interpréter ce récit : son regard se pose successivement sur tous les soldats tâchés par la boue et sur Hirose. Il fait du récit de cet individu particulier l'explication des traces que porte tout le groupe. Nous sommes tenté de voir dans cette lecture du récit d'un authentique soldat par un autre soldat authentique un modèle auquel Hino invite son lecteur, qui valide l'idée d'un va-et-vient entre la valeur individuelle et la valeur collective de l'expérience de guerre, et dont l'aboutissement est par ailleurs donné de façon ostentatoire : il s'agit de voir la noblesse dans la boue.

L'image est cependant plus riche qu'il n'y paraît : ce n'est qu'en écoutant le récit de Hirose que la crasse est interprétée comme une marque de " noblesse ». Les apparences se sont jouées de Hino comme du lecteur qui ont tous deux glissé sur la première mention de ces soldats qui « étaient 
trempés de sueur et portaient des uniformes maculés de terre rouge ». Cette crasse est le signe d'une histoire qui doit être racontée, elle signifie en dernière instance que tous les soldats méritent d'être re-regardés, que c'est le rôle de la parole - individuelle cette fois-ci, celle de Hirose, celle de Hino - de rendre leur sens particulier à ces clichés dans le piège desquels Hino lui-même a pu tomber.

\section{Conclusion}

Mugi to heitai est le lieu d'une tension entre deux genres littéraires, chacun porteur de valeurs a priori opposées : en tant que récit personnel, le texte doit montrer qu'il se fonde sur une expérience vécue, garante de la crédibilité du propos; en tant que récit de guerre, il se doit en revanche de dépasser ce caractère individuel pour dépeindre la guerre en tant qu'expérience commune. Nous avons ici tenté de montrer que le lien entre l'expérience de Hino et cette expérience commune ne va pas de soi. Il est au contraire l'objet d'une mise en scène complexe, laquelle repose d'une part sur une écriture qui revendique sa transparence, allant jusqu'à s'assimiler à une reproduction brute du réel, et d'autre part sur des procédés littéraires permettant d'intégrer à la parole de Hino la parole des autres soldats. La biographie de Hino a évidemment joué un rôle dans la valeur qui fut attribuée à ses textes pendant la guerre, mais il nous apparaît dans Mugi to heitai comme un porte-parole dont l'autorité découle du texte lui-même.

En somme, le statut de Hino pendant la guerre ne lui assurait pas en tant que tel d'autre valeur que celle de témoin à une période où, comme nous l'avons déjà dit, autorités et éditeurs faisaient paraître quantité de témoignages (ceux des écrivains envoyés sur le front, ceux des soldats dont on publiait les journaux, ceux des journalistes). Il nous semble à ce titre que ce qui permit à Mugi to heitai de n'être pas un témoignage parmi d'autres et de connaître un tel succès est bien ce statut de porte-parole que l'auteur se fabrique au cours du texte. Les processus rhétoriques à l'œuvre ici permirent à Hino de prétendre à un rôle public qu'un "simple " témoignage n'auraient probablement pas permis de garantir. Cette figure publique répondit ainsi parfaitement aux désirs de l'armée en termes de propagande : cette voix des soldats que Hino donnait à entendre à la population civile était sous contrôle direct du service d'Information de l'armée. 
Du point de vue du lecteur contemporain, le fait que Mugi to heitai ait fait partie du dispositif de propagande mis en place par les autorités pendant la guerre rend plus légitime, ou du moins plus évidente, la tentation de chercher à mettre au jour les mécanismes qui ont permis au texte d'être perçu comme un témoignage ; il serait aujourd'hui très facile d'en pointer les mensonges et les fondements idéologiques. Ne considérer Mugi to heitai que comme un cas d'école de la propagande de guerre nous semble cependant par trop réducteur : la littérature refuse toujours de n'être que « de la littérature " au sens vulgaire de l'expression consacrée, et la revendication au sein d'un texte d'une valeur qui le dépasse n'est certainement pas l'apanage de la littérature de propagande. Il n'est que de songer à la littérature de guerre écrite après la défaite : un auteur tel qu'Ōoka Shōhei 大岡昇平 ne prétend évidemment pas non plus ne parler que de lui. 


\section{Bibliographie}

\section{BARTHES Roland, 1953}

Le degré zéro de l'écriture, suivi de Nouveaux essais critiques, Paris, Seuil.

BEAUPRÉ Nicolas, 2006

Écrire en guerre, écrire la guerre. France, Allemagne 1914-1920, Paris, CNRS éditions.

\section{BRAUD Michel, 2006}

La forme des jours. Pour une poétique du journal personnel, Paris, Seuil.

Bungei 文藝, 1938

Numéro d'octobre, 6 (10).

FOUCAULT Michel, 2001

"Qu'est-ce qu'un auteur?", Dits et écrits I, Paris, Gallimard.

FURUYA Tsunatake 古谷綱竹, 1939

《Hino Ashihei ron 》 火野蔁平論

(Sur Hino Ashihei), Chūō kōron

中央公論, juillet, 54 (7) : 266-277.

\section{HIBINO Shirō 日比野士郎, 1940}

«Hino Ashihei to Ueda Hiroshi »

火野蔁平と上田廣 (Hino Ashihei et Ueda Hiroshi), Bungei 文藝, janvier, 8 (1) : 80-87.

HINO Ashihei 火野蔁平, 1938 Mugi to heitai. Joshū kaisen jūgunki 麦と兵隊——徐州会戦従軍記 (Les Blés et les Soldats. Carnet de campagne de la bataille de Xuzhou), Tokyo, Kaizōsha 改造社.
HINO Ashihei, 1958

« Kaisetsu » 解説 (Commentaire), Hino Ashihei senshū 火野蔁平選集 (Anthologie de Hino Ashihei), vol. 2, Tokyo, Sōgensha 創元社.

ITŌ Sei 伊藤整, 1944

Sensō no bungaku 戦争の文学 (La littérature de la guerre), Osaka, Zenkoku shobō 全國書房.

IKEDA Hiroshi 池田浩士, 2000 Hino Ashihei ron 火野蔁平論 (Sur Hino Ashihei), Tokyo, Inpakuto shuppankai インパクト出版会.

\section{KAEMPFER Jean, 1998}

Poétique du récit de guerre, Paris, José Corti.

KITAGAWA Kenzō 北河賢三, 2003

Sensō to chishikijin 戦争と知識人 (La guerre et les intellectuels), Tokyo, Yamakawa shuppansha 山川出版社.

\section{KUSHNER Barak, 2006}

The Thought War. Japanese Imperial Propaganda, Honolulu, University of Hawai'i Press.

MIYOSHI Tatsuji 三好達治, 1938

«Mugi to heitai no kansō» 麦と兵隊の 感想 (Pensées sur Les Blés et les Soldats), Bungei 文藝, septembre, 6 (9) : 219-222. 
94 | Guillaume MULLER | L'ambivalence du récit de guerre

ROSENFELD David M., 2002

Unhappy Soldier. Hino Ashihei and

Japanese World War Literature, Lanham,

Lexington Books.

SUGIYAMA Heisuke 杉山平助, 1939

《Hino Ashihei ron » 火野蔁平論 (Sur

Hino Ashihei), Kaizó 改造, octobre,

21 (10) : 300-314. 